Скопје, Македонија

\title{
A STATEMENT OF DIFFERENTIAL CALCULUS IN CONTEXT OF CODOMAIN OF THE FUNCTION
}

\author{
JOVAN V. MALEŠEVIĆ
}

\begin{abstract}
In this article we give an analogue of Statement 3 from [1] with appropriate geometrical interpretation in sense of codomain (image) of the function $f(x)$.
\end{abstract}

Using theorem of Darboux, some generalizations and analogizes of theorems D. Trahan [2] and T. Flett [4] by Theorem 3 from [1] are given. In this article using Lemma 2 [2] of D. Trahan and Corollary 3 from [3], the following Statement, with the mentioned emphasis on the geometrical interpretation, is given.

Statement. Let $f:[a, b] \longrightarrow R$ be differentiable function. If there exists a point $x_{0} \in(a, b)$, such that $f^{\prime \prime}\left(x_{0}\right)$ exists and

$$
\left(f^{\prime}(r)-\frac{f(r)-f\left(x_{0}\right)}{r-x_{0}}\right)\left(\frac{f(b)-f(a)}{b-a}-\frac{f(r)-f\left(x_{0}\right)}{r-x_{0}}\right)>0, \text { for } r=a \text { and } r=b,
$$

then exist at least two values $\xi \in(a, b)$ such that

$$
f^{\prime}\left(\xi_{i}\right)=\frac{f\left(\xi_{i}\right)-f\left(x_{0}\right)}{\xi_{i}-x_{0}}, \quad i=1,2 .
$$

Proof. Let us introduce function

$$
F(x)=\left\{\begin{array}{cl}
\frac{f(x)-f\left(x_{0}\right)}{x-x_{0}} & : x \neq x_{0} \in(a, b) \\
f^{\prime}\left(x_{0}\right) & : x=x_{0}
\end{array}\right.
$$

It is true, for $x \neq x_{0}$ :

$$
F^{\prime}(x)=\frac{f^{\prime}(x)\left(x-x_{0}\right)-\left(f(x)-f\left(x_{0}\right)\right)}{\left(x-x_{0}\right)^{2}}
$$


and

$$
\begin{aligned}
\lim _{x \rightarrow x_{0}} \frac{F(x)-F\left(x_{0}\right)}{x-x_{0}} & =\lim _{x \rightarrow x_{0}} \frac{\frac{f(x)-f\left(x_{0}\right)}{x-x_{0}}-f^{\prime}\left(x_{0}\right)}{x-x_{0}}=\lim _{x \rightarrow x_{0}} \frac{f^{\prime}(x)-f^{\prime}\left(x_{0}\right)}{2\left(x-x_{0}\right)} \\
& =\lim _{x \rightarrow x_{0}} \frac{f^{\prime \prime}(x)}{2}=\frac{f^{\prime \prime}\left(x_{0}\right)}{2}
\end{aligned}
$$

ie. function $F(x)$ is differentiable over segment $[a, b]$ and $F^{\prime}\left(x_{0}\right)=\frac{f^{\prime \prime}\left(x_{0}\right)}{2}$.

$\mathbf{1}^{\mathbf{0}}$. Based on the sign of the difference $\frac{f(b)-f(a)}{b-a}-\frac{f(r)-f\left(x_{0}\right)}{r-x_{0}} \underset{(<)}{>} 0$, in $r=a$, we obtained one upper (lower) bound for value $F(a)$ as follows

$$
F(a)=\frac{f(a)-f\left(x_{0}\right)}{a-x_{0}} \underset{(>)}{<} \frac{f(b)-f(a)}{b-a} .
$$

Previous inequality is equivalent with

$$
(f(b)-f(a))\left(a-b+b-x_{0}\right) \underset{(>)}{<}\left(f(a)-f(b)+f(b)-f\left(x_{0}\right)\right)(b-a),
$$

ie.

$$
(f(b)-f(a))\left(b-x_{0}\right) \underset{(>)}{<}\left(f(b)-f\left(x_{0}\right)\right)(b-a) .
$$

meaning that

$$
\frac{f(b)-f(a)}{b-a} \underset{(>)}{<} \frac{f(b)-f\left(x_{0}\right)}{b-x_{0}}=F(b) ;
$$

and finally

$$
F(a)=\frac{f(a)-f\left(x_{0}\right)}{a-x_{0}} \underset{(>)}{<} \frac{f(b)-f(a)}{b-a} \underset{(>)}{<} \frac{f(b)-f\left(x_{0}\right)}{b-x_{0}}=F(b) .
$$

Therefore

$$
F(a) \underset{(>)}{<} F(b) \Longleftrightarrow(F(b)-F(a)) \underset{(<)}{>} 0
$$

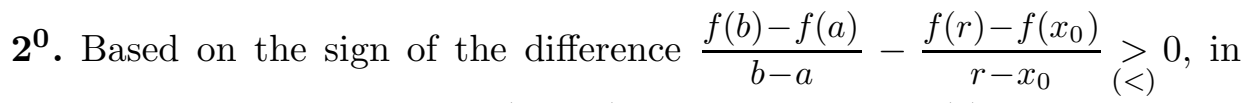
$r=b$, we obtained one lower (upper) bound for value $F(b)$ as follows

$$
\frac{f(b)-f(a)}{b-a} \underset{(<)}{>} \frac{f(b)-f\left(x_{0}\right)}{b-x_{0}}=F(b),
$$

Previous inequality is equivalent with

$$
(f(b)-f(a))\left(b-a+a-x_{0}\right) \underset{(<)}{>}\left(f(b)-f(a)+f(a)-f\left(x_{0}\right)\right)(b-a),
$$


ie.

$$
(f(b)-f(a))\left(x_{0}-a\right) \underset{(>)}{<}\left(f\left(x_{0}\right)-f(a)\right)(b-a) .
$$

meaning that

$$
F(a)=\frac{f(b)-f(a)}{b-a} \underset{(>)}{<} \frac{f(b)-f\left(x_{0}\right)}{b-x_{0}}=F(b)
$$

and finally

$$
F(a)=\frac{f(a)-f\left(x_{0}\right)}{a-x_{0}} \underset{(<)}{>} \frac{f(b)-f(a)}{b-a} \underset{(<)}{>} \frac{f(b)-f\left(x_{0}\right)}{b-x_{0}}=F(b) .
$$

Therefore

$$
F(a) \underset{(<)}{>} F(b) \Longleftrightarrow(F(b)-F(a)) \underset{(>)}{<0 .}
$$


$\mathbf{3}^{\mathbf{0}}$. Based on the sign of the difference $f^{\prime}(r)-\frac{f(r)-f\left(x_{0}\right)}{r-x_{0}} \underset{(<)}{>} 0$, in $r=a$, we obtained

$$
\begin{aligned}
F^{\prime}(a) & =\left.\frac{f^{\prime}(x)\left(x-x_{0}\right)-\left(f(x)-f\left(x_{0}\right)\right)}{\left(x-x_{0}\right)^{2}}\right|_{x=a} \\
& =\frac{f^{\prime}(a)\left(a-x_{0}\right)-\left(f(a)-f\left(x_{0}\right)\right)}{\left(a-x_{0}\right)^{2}} \\
& =\frac{1}{a-x_{0}}\left(f^{\prime}(a)-\frac{f(a)-f\left(x_{0}\right)}{a-x_{0}}\right) \underset{(>)}{<0,}
\end{aligned}
$$

ie.

$$
F^{\prime}(a) \underset{(>)}{<0}
$$

Analogously, in $r=b$, we obtained

$$
F^{\prime}(b)=\frac{1}{b-x_{0}}\left(f^{\prime}(b)-\frac{f(b)-f\left(x_{0}\right)}{b-x_{0}}\right) \underset{(<)}{>0} 0
$$

ie.

$$
F^{\prime}(b) \underset{(<)}{>0} 0
$$

$\mathbf{4}^{\mathbf{0}}$. From (6) and (9) follows

$$
F^{\prime}(a)(F(b)-F(a))<0,
$$

and analogously, based on (8) and (10), we can conclude

$$
F^{\prime}(b)(F(b)-F(a))<0 .
$$

Therefore, is true

$$
F^{\prime}(a)(F(b)-F(a))<0 \wedge F^{\prime}(b)(F(b)-F(a))<0 .
$$

Next, using Lemma 2 [2] and Corollary 3 [3] respectively, existence least two $\xi \in(a, b)$ follows such that $F^{\prime}(\xi)=0$ and therefore follows (in Fletts denotation):

$$
\left(\exists \xi_{i} \in(a, b)\right) f^{\prime}\left(\xi_{i}\right)=\frac{f\left(\xi_{i}\right)-f\left(x_{0}\right)}{\xi_{i}-x_{0}}, \quad i=1,2 .
$$

Geometrically interpretation. In this part we geometrically determined part of codomain of the function $f$ over segment $[a, b]$ in case of tangents in points $A$ and $B$ and their intersection $\left(f^{\prime}(a) \neq f^{\prime}(b)\right)$, based on the following conditions:

$$
f^{\prime}(a)-\frac{f\left(x_{0}\right)-f(a)}{x_{0}-a}>0 \wedge f^{\prime}(b)-\frac{f(b)-f\left(x_{0}\right)}{b-x_{0}}>0 .
$$


¿From previous proof of the Statement we have:

ie.

$$
\frac{f\left(x_{0}\right)-f(a)}{x_{0}-a}<f^{\prime}(a) \wedge \quad f^{\prime}(b)>\frac{f(b)-f\left(x_{0}\right)}{b-x_{0}},
$$

(16)

$f\left(x_{0}\right)<T_{A}: f\left(x_{0}\right)<f(a)+f^{\prime}(a)\left(x_{0}-a\right) \wedge f\left(x_{0}\right)>T_{B}: f\left(x_{0}\right)>f(b)+f^{\prime}(b)\left(x_{0}-b\right) ;$

therefore we have the following Figure of function $f(x)$ over segment $[a, b]$ and tangent in point $A$ (bellow this tangent is region $D_{1}$ ), and tangent in point $B$ (above this tangent is region $D_{2}$ ). On this way in Figure we have interior region $D=D_{1} \cap D_{2}$ for both possibility in (14). Special consideration in connection of codomain of the function $f(x)$ in regions $D_{1}$ and $D_{2}$ correspond relations (11) and (12) respectively.

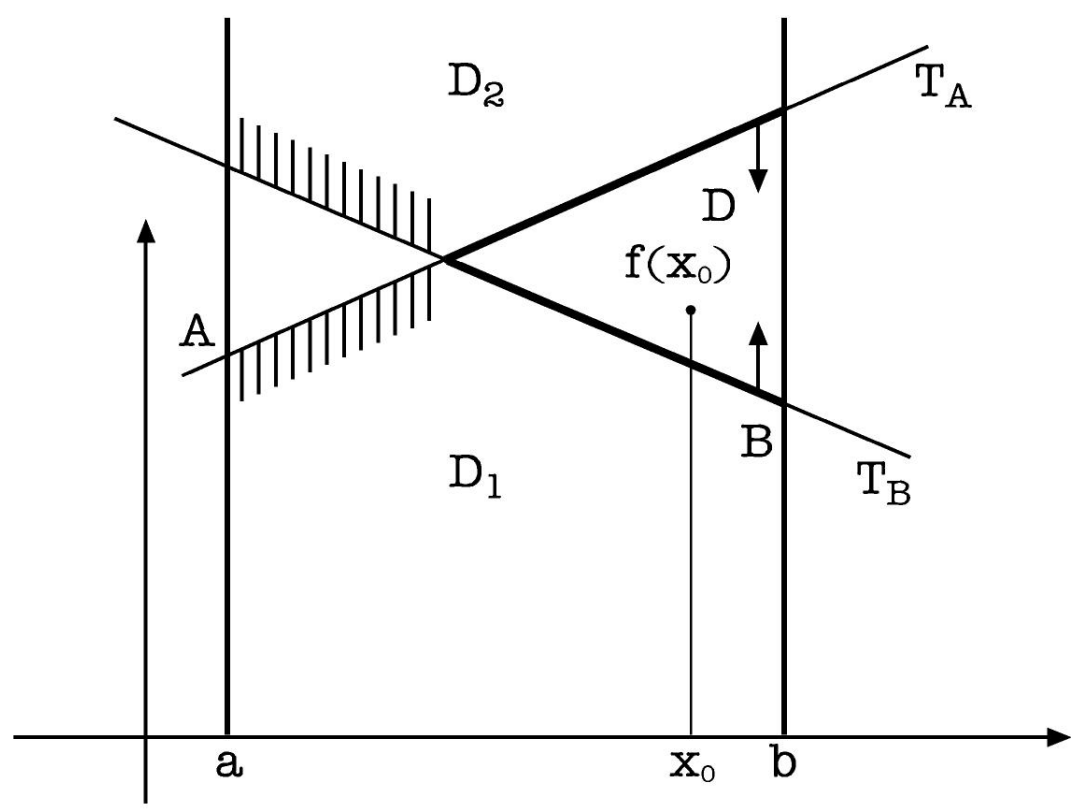

Figure

\section{REFERENCES}

[1.] J.V. Malešević: Jedna generalizacija formule Tejlora, Matematički vesnik, 12 (27), 1975, 375-384.

[2.] D.H. Trahan: A new type of mean values, Mat. Magazine 1966. 39, No5, 264-266.

[3.] J.V. Malešević: O jednoj teoremi G. Darboux-a i teoremi D. Trahan-a, Matematički vesnik, 4 (17), 1980, 32.

[4.] T.H. Flett: A mean value theorem, Mat. Gazette, 1958, 42, 38-39. 


\title{
ЕДНО ТВРДЕЊЕ ЗА ДИФЕРЕНЦИЈАЛНОТО СМЕТАҢЕ ВО
} КОНТЕКСТ НА КОДОМЕНОТ НА ФУНКЦИЈАТА

\author{
Јован Малешевиќ
}

Р е зи м е

Во овој труд даваме аналогно тврдење на тврдењето 3 од [1] со соодветна геометриска интерпретација во смисла на кодоменот (сликата) на функцијата $f(x)$.

FutošKa 60, Novi SAD, SERBia

E-mail address: malesh@eunet.rs 